\title{
Deep Neural Networks for Future Low Carbon Energy Technologies: Potential, Challenges and Economic Development
}

\author{
Rameez Asif \\ Department of Electronics and Electrical Engineering, University of Strathclyde, Glasgow, UK \\ Power Networks Demonstration Centre (PNDC), University of Strathclyde, Glasgow, UK \\ Corresponding Author: rameez.asif@strath.ac.uk
}

\begin{abstract}
The global energy demands are growing every year, and fossil fuels won't be able to fulfill our energy needs in the near future. Carbon emissions from the fossil fuels hit an all-time high in 2018 due to increased energy consumption around the globe. On the other hand, renewable energy is an emerging technology and considered as a reliable alternative to the fossil fuels. It is much safer and cleaner than conventional sources. With the advancements in technology, the renewable energy sector has made significant progress in the last decade. One most significant challenge, the large scale renewable energy farms are facing is the un-predictability of the weather patterns. This stochastic nature of the weather data is impacting the solar and wind farms significantly. Although, the classical technologies are in place for weather forecasting but they are not efficient enough to give a feedback to thebasestation for any sudden change or future predictions. The demand for renewable energy will only increase in the future. And, that is why renewable energy companies need to invest in Artificial Intelligence (AI), Internet-of-Things (IoT), and other emerging technologies to improve productivity and overcome the shortfalls. Even the large consumers of renewable energy, like supermarkets, factories, offices, railways can use AI technology to make data-driven decisions on power usage and demand. In this article, we present an overview of AI techniques for modelling, prediction and forecasting of wind farming data. Additionally, we have presented economic impact of low carbon energy techniques by analysing the climate change patterns and diverse sources of power generation for the Scotland, United Kingdom region, as a case study.
\end{abstract}

\section{INTRODUCTION}

Motivated by the recent technological advancements and increasing concern over the sustainability and environmental impact of conventional fossil fuel usage, the likelihood of producing clean, sustainable power in substantial quantities from renewable energy sources triggered interest around the globe [1], [2]. The power distribution grid is one of the complex networks on Earth, but it is evolving rapidly with the addition of variable low carbon energy sources. Due to the inherent stochastic nature of the wind and solar, the current grid faces many challenges in accommodating the diversity of renewable energy [3]. The utility industry needs digital smart systems that can help improve the integration of renewable into the existing grid and make renewable energy an equal player in the energy supply. That is the reason, AI technology can improve the reliability of renewable energy and modernize the overall grid [4]. The energy grid can be interconnected with devices and IoT sensors to collect a large amount of data that can be coupled with the cloud servers for real-time storage [5]. When integrated with AI, this data can give new insights to the grid operators for better control operations. It offers flexibility to the energy suppliers to cleverly adjust the supply with demand. Additionally, machine-to-machine (M2M) communications and advanced IoT sensors can make weather and load predictions that can overall improve the integration and efficiency of renewable energy sector. AI can help with the integration of microgrids and managing distributed energy. When the renewable energy generated power is added to the primary grid, it becomes hard to balance the energy flow with-in the grid [6]. The AI-powered control system can play a vital role in solving the quality and congestion issues especially for the next generation digital sub-station scenarios. The AIpowered predictive analysis can predict the future weather and wind patterns but also collect the data from wind turbine sensors to monitor wear and tear. The system will monitor the overall health of the equipment and alert the operator when the maintenance is needed [7].

\section{ECONOMIC IMPACT OF LOW-CARBON ENERGY}

Climate change has been seen as an emerging global affair and is the defining issue of our generation [8]. From the changing weather patterns [9], that are causing long term damage to the food production, catastrophic flooding, rising of sea beds, melting of the northern glaciers, damaging the ozone layer, it is a well known fact that the impacts of climate change are wide ranging and unprecedented in scale [10]. Greenhouse gases are instinctive in nature and are essential for the survival of living beings [11], by keeping some of the solar warmth from reflecting back into space and making earth livable. But after exponential increase in the industrialization, deforestation, and large scale agriculture, quantities of greenhouse gases in the atmosphere have risen to the record levels in recent centuries [12]. As populations, economies and standards of living grow, so does the aggregate level of greenhouse gas (GHGs) emissions.

Due to the research data collected from the observatories around the world [13], it is a well-established fact that the average increase in the temperatures is due to the burning of fossil fuels [14] that are indirectly linked to the economic development indicators [15]. These facts are:

1) The concentration of GHGs in the earth's atmosphere due to the fuels is directly linked to the average global 

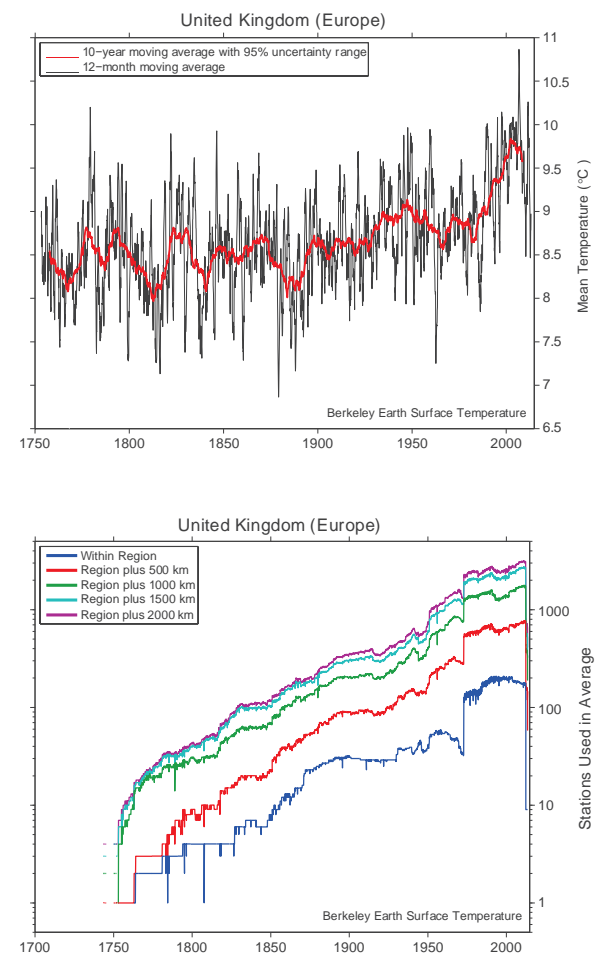

Fig. 1. Analysis of the output data of: (top) regional average air temperatures over land and change to GHG and (below) number of weather stations used for data processing (monthly mean observations:15M)

temperature on the earth.

2) The concentration has been rising steadily, and mean global temperatures along with it, since the time of the industrial revolution and economic development.

3) The most abundant GHGs, accounting for about twothirds of GHGs, carbon dioxide $\left(\mathrm{CO}_{2}\right)$, is largely the product of burning fossil fuels and not using the renewable energy resource.

In order to give a comprehensive assessment of the rise of average temperatures over the last 3 centuries due to the cumulative $\mathrm{CO} 2$ emissions, we analyzed the data ranging from the year 1750 till $2013^{1}$. From the data, as depicted in Fig. 1 and 2, the average temperature increase in the United Kingdom (UK) is approximately $+2.3 \mathrm{deg}-\mathrm{C}$ in 100 years time and that can be linked with the massive industrialization. Global warming [16] mitigation requires rapid, far-reaching and unprecedented changes in all aspects of economy, industry and society. The two well-known conventions, i.e. Kyoto Protocol [17] and Paris Agreement [18], stipulates that the targets to reduce the greenhouse emissions are strengthen over time, both in ambition and scope due to the general trends in research and development of renewable (low carbon) energy resources [19].

Promotion and investment [20] in low carbon and related technologies by the governments is a positive way forward.

${ }^{1}$ Source of Data Sets: Berkeley Earth Library (Climate Science and Strategic Analysis), www.berkeleyearth.lbl.gov
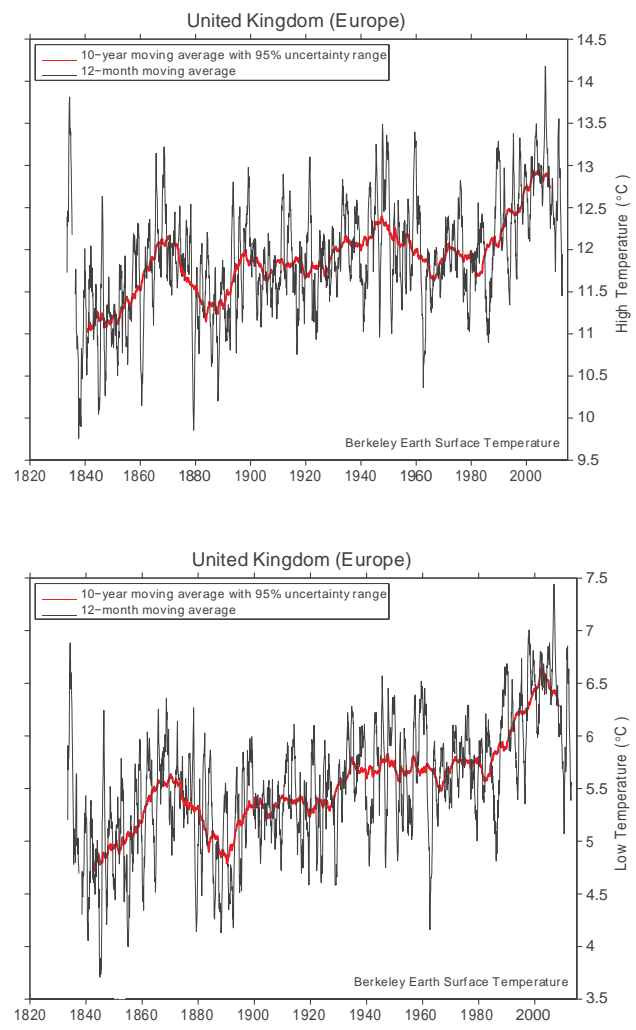

Fig. 2. Analysis of the output data of: (top) regional mean of daily high temperatures and (below) regional mean of daily low temperatures (monthly mean observations: $15 \mathrm{M})$

Already massive subsidies [21], to foster the low carbon technologies is having major impact on energy prices, fuel poverty, off-shore and on-shore wind farming, bio-fuels, electric vehicles etc. For elaboration, we take the case-study of wind farming in the Scotland region as compared to the rest of the United Kingdom that is the major share of low carbon energy in this region, as depicted in Fig. 3. On-shore wind farming are at or near the point where they can supply electricity to the grid without subsidy. But one of the key factors that hinders new developments is uncertainty about the costs and installation of the projects with respect to the energy generated and supply to the domestic consumers, as in Fig. 4. These statistics, clearly depicts the demand, generation through renewables and storage. This emphasize and translates into the data-driven approach to efficiently and intelligently harvest the low carbon energy [22]. The data in Fig. 4 depicts the time-line graph for the grid in Scotland, United kingdom. The data is normalised and modelled in proportion to the wind power and demand data ${ }^{2}$.

To meet future usage for clean and sustainable energy, tremendous progress has been achieved in development for scavenging wind energy [23]. Most recently, the researchers have developed advanced harvesting techniques, such as triboelectric nano-generators to enhanced the generation of

${ }^{2}$ Source of Data Sets: UK National Grid Status, Scottish Scientists. 


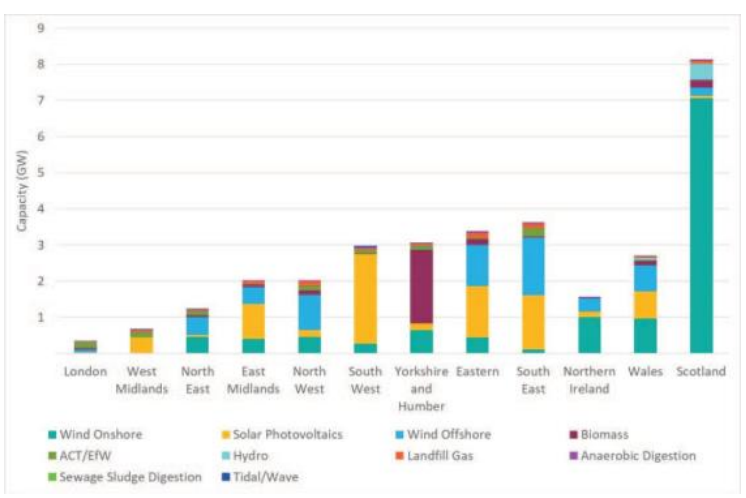

Fig. 3. Deployment of Renewable Energy Technologies as at the end of 2017. (Statistics: BEIS Renewable Energy Planning Database (REPD))

energy from wind farms. Despite of the hardware advancements, the vital challenge of adapting wind farming on large scale is the control of its un-certain output. The efficacious solution to this problem is the precise estimate of the future wind power mainly dependent on the weather patterns, i.e. data analytics. The correct Wind Power Forecasting (WPF) helps in improving the operation scheduling of power systems. The operating schedule for backup generators and storage systems are optimized based on the accurate WPF [24].

\section{DEEP NEURAL NETWORKS FOR LOW CARBON ENERGY}

It is widely accepted that accurate WPF significantly reduces the risks of incorporating wind power in power distribution networks [25]. Generally, the WPF results are in the deterministic form (i.e., point forecasting). Reducing the forecasting errors of WPF is the focus of many researchers [26]. A point forecasting is the estimated value of future wind energy generation. However, wind power generation is a random variable having a Probability Density Function (PDF), and point forecasts are unable to capture the uncertainty of this random variable. This is the limitation of the point forecasts. Therefore, point forecasts have limited use in stability and security analysis of power systems [27] especially for the low carbon energy technologies. To overcome the limitation of point forecasts, artificial intelligence (AI) or Deep Learning Methods (DLM) are widely used in the field of WPF. Deep Neural Networks (DNN) have the inherent property of automatic modeling of the wind power characteristics [28]. The energy data collected and modelled with high accuracy proved to be a useful resource for wind power predictive analytics. Recently, big data driven models show significant accuracy in wind power forecasting, and the most impactful examples are Google Deep Mind Project [29], Australia's First AI based Wind Farm [30] and Makani Shell Wind Kites Project [31].

\section{A. Google DeepMind Wind Farm Project}

In early 2019, Google and DeepMind started a project for on-shore wind farms, where machine learning (ML) algorithms are applied to a 700 megawatts facility located in central United States. These wind farms are a part of Google's global fleet of renewable energy projects [32]. Using a deep neural network trained on widely available weather forecasts and historical turbine data, the researchers configured the DeepMind system [33] to predict wind power output 36 hours in-advance of actual generation, as predicted in Fig. 5. Based on these predictions, their model recommends how to make optimal hourly delivery commitments to the power grid a full day in advance. This is important, because energy sources that can be scheduled (i.e. can deliver a set amount of electricity at a set time) are often more valuable to the power grid. ${ }^{3}$

Based on these algorithms, the Google has announced, they have boosted the value of wind generation by approximately $20 \%$. Moreover, by reducing the variability of wind power, the renewable energy source becomes sufficiently more predictable and valuable, giving wind farm operators more data-driven assessments of how to meet upcoming electricity demand, i.e. intelligent wind farming.

\section{B. Australia's First AI Wind Farm Project}

In July 2019, Australia launched it's first wind farm equipped with AI software developed by Advanced Microgrid Solutions (AMS) [34]. AI software gives the researchers an edge to manage risk and improve margins in energy markets that are becoming more challenging with high penetration of renewable generation. Furthermore, the researchers have trained several hundred variations of the deep neural network model on energy generation and weather data.

\section{Makani Shell Wind Kites Project}

Makani Shell Wind Kites have the potential to accelerate access to new offshore wind areas and complement the expansion of low carbon industry ecosystem. Unlike most floating offshore wind technologies deployed at water depths greater than 50 meters, Makani's kites don't need to be supported by large complex platforms. Hence reducing the cost of regular maintenance and teleprotection [31]. Instead, the researchers plan to mount Makani's kite on a small spar buoy moored with a synthetic line and gravity anchor. This is possible because Makani's kites are 90\% lighter than turbines of a similar power rating and the overall system is smaller, replacing tons of steel with lightweight electronics and smart intelligent software, which reduces overall costs of wind energy generation. This project also incorporates AI techniques to predict wind turbine failures, AI-managed radio to efficiently harness the wind energy.

\section{INTELLIGENT WIND SPEED FORECASTING (I-WSF)}

Wind speed forecasting (WSF) is a very important parameter for large-scale wind farms particularly due to the cost related issues, dispatch planning and energy market operations [35], [36]. These AI based algorithms are employed for optimal operational policies and operating cost management [37], [38], load balancing [35], [39], site and

\footnotetext{
${ }^{3}$ Source of Data: Google and DeepMind.
} 


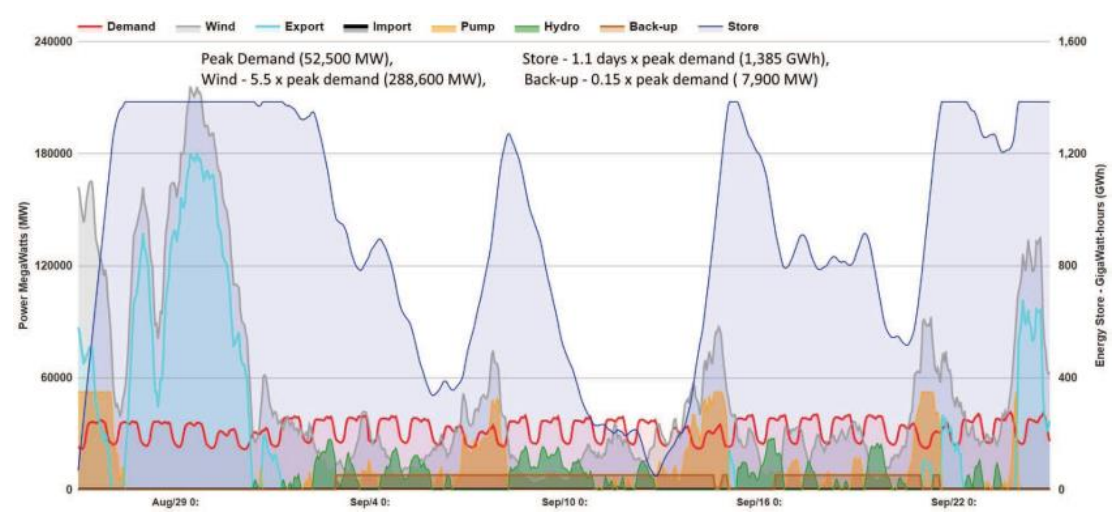

Fig. 4. Low carbon energy generation and storage in Scotland, United Kingdom (Peak Demand (52,500 MW), Store - 1.1 days x peak demand (1,385 GWh), Wind - 5.5 x peak demand (288,600 MW), Back-up - 0.15 x peak demand ( 7,900 MW))

capacity planning [40], [41] and on-grid contribution of the energy with respect to the market trends [35]-[37]. Several heuristic approaches had been adopted to get the maximum power generation based on the measure of the nominal power and historical atmospheric data [42], [43]. While classical approach may help you to forecast energy demands and consumption but inaccurate WSF will become a potential point of failure of large scale on-shore and offshore wind farms [44]. Recently, data mining and AI based forecasting algorithms have been applied to the wind farming.By making use of both satellite data from weather forecasts and ML algorithms trained to analyze the industry's data to make more accurate forecasting. The high-resolution weather forecasting is generated from satellite images. These images are then used to generate both large-scale and smallscale weather models. The ML algorithms analyze such big data and predicts the state of the atmosphere in a particular region. Support Vector Regression (SVR), is one of the AI algorithm, that has been used for prediction of wind speed and other atmospheric variables with positive results [45][49]. Before going in much details of SVR, here are the few important terms:

- Kernal: The function used to map a lower dimensional data into a higher dimensional data.

- Hyper Plane: The line that will will help us predict the continuous value or target value.

- Boundary Line: There are two lines other than Hyper Plane which creates a margin. The support vectors can

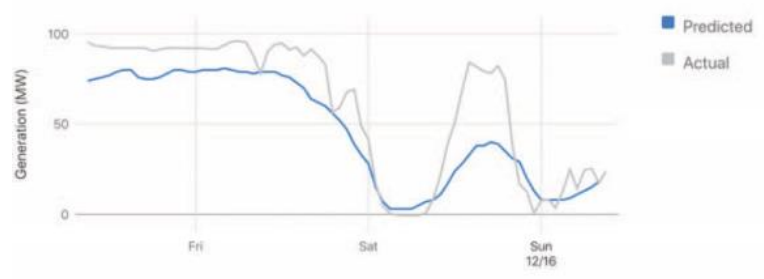

Fig. 5. Deep Neural Network (DNN) trained to make a model based on weather predictions for wind farms 36 hours in advance [32] be on the Boundary lines or outside it. This boundary line separates the two classes.

- Support Vectors: These are the data points which are closest to the boundary. The distance of the points is minimum or least.

SVR is considered as a non-parametric method because it mainly relies on the kernel functions. For simplification, Support Vector Machine (SVM) can also be used as a regression method, maintaining all the main features that characterize the algorithm (maximal margin). The SVR uses the same set of principles as the SVM for classification, with only a few minor differences. First of all, because output is a real number it becomes very difficult to predict the information at hand, which has infinite possibilities. In the case of regression, a margin of tolerance (epsilon) is set in approximation to the SVM which would have already requested from the problem [50]. But besides this fact, there is also a more complicated reason, the algorithm is more complicated therefore to be taken in consideration. However, the basics are always the same: to minimize error ratio, individualizing the hyper plane which maximizes the margins, while keeping in mind that part of the error is tolerated, as depicted in Fig. 6. Mathematically, the linear SVR can be given as in Eq. 1. Basically, we are trying to decide a decision boundary at ' $\varepsilon$ ' distance from the original hyper plane such that data points closest to the hyper plane or the support vectors are within that boundary line. Thus the decision boundary is our margin of tolerance, i.e. we are going to take only those points those are within this boundary.

$$
y=\sum_{N}^{i=1}\left(\alpha-\alpha^{*}\right) \cdot * x_{i}, x+b
$$

Whereas, for non-linear SVR methods, the kernel functions transform the data into a higher dimensional feature space to make it possible to perform the linear separation, as depicted in Fig. 7.

Mathematically, this can be expressed as in Eq. 2 and 3. 

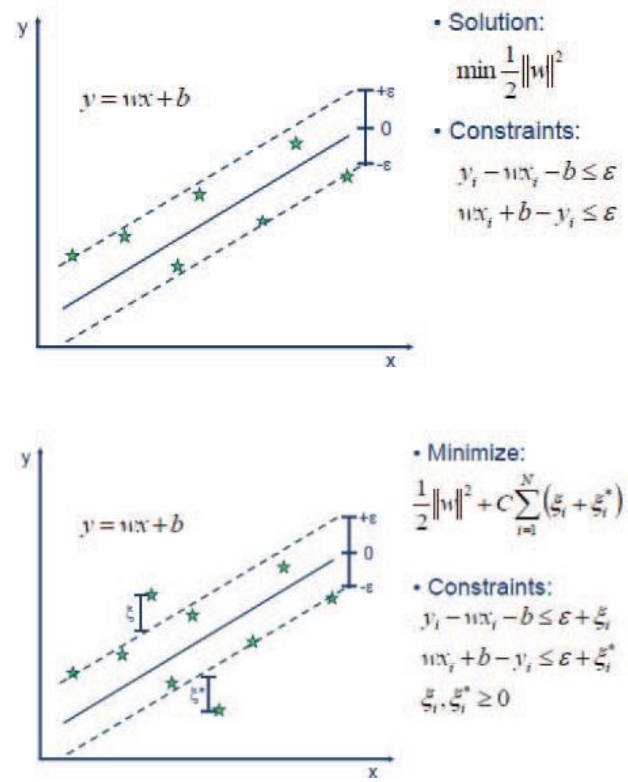

Fig. 6. Schematic of the one-dimensional linear support vector regression (SVR) model.

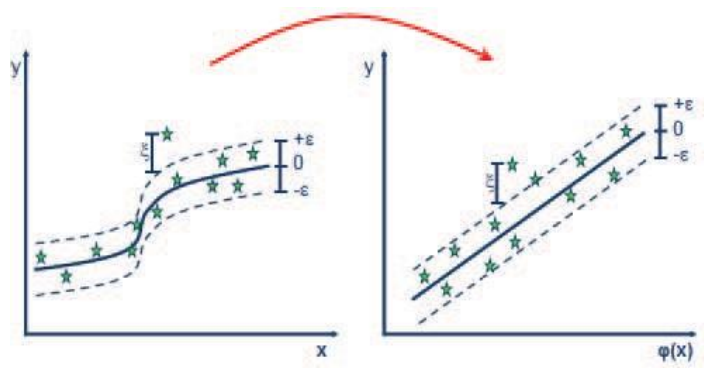

Fig. 7. Schematic of the one-dimensional non-linear support vector regression (SVR) model.

$$
\begin{gathered}
y=\sum_{N}^{i=1}\left(\alpha-\mathbf{\alpha}^{*}\right) \cdot * \phi\left(x_{i}\right), \phi(x)+b \\
y=\sum_{N}^{i=1}\left(\alpha-\alpha^{*}\right) \cdot k\left(x_{i}, x\right)+b
\end{gathered}
$$

Whereas, the Kernel functions used to solve the above equations, i.e. the polynomial and Gaussian basis function, are given as in Eq. 4 and 5, respectively:

$$
\begin{array}{r}
k\left(x_{i}, x\right)=\left(x_{i} \cdot x_{j}\right)^{d} \\
\mathrm{I} \mathrm{I}_{x_{i}-x_{j}} \overline{\mathrm{I}}_{2} \\
2 \sigma^{2}
\end{array}
$$

\section{Future Potential AND Challenges}

Integration of artificial intelligence (AI) with classical wind/solar farms and Intelligent Energy Storage (IES) applications can provide a sustainable and reliable solution to the next generation renewable energy industry. With the help of advancements in the IoT infrastructure, smart grid will be able to analyze a vast amount of data collected from several sensors and make timely decisions on energy generation and allocation. The main challenge is to harness AI to improve the efficiency of wind and solar by using deep neural networks to enhance predictability of generation and grid stability. For this, industry have to adopt the latest trends, install new hardware that is capable of running AI algorithms, integrate AI in classical systems and have unified cyber-security standards.

\section{ACKNOWLEDGMENT}

We would also like to say thanks to Dr. Kinan Ghanem and Dr. Federico Coffele from Power Networks Demonstration Centre (PNDC) for useful discussions on smart grids, renewable energy resources and commercial developments.

\section{REFERENCES}

[1] J. A. Turner, "A realizable renewable energy future," Science, vol. 285, no. 5428, pp. 687-689, 1999. [Online]. Available: https://science.sciencemag.org/content/285/5428/687

[2] I. Dincer, "Renewable energy and sustainable development: a crucial review," Renewable and Sustainable Energy Reviews, vol. 4, no. 2, pp. 157 - 175, 2000. [Online]. Available: http://www.sciencedirect.com/science/article/pii/S1364032199000118

[3] Q. Yang, J. A. Barria, and T. C. Green, "Communication infrastructures for distributed control of power distribution networks," IEEE Transactions on Industrial Informatics, vol. 7, no. 2, pp. 316-327, May 2011.

[4] P. S. Georgilakis and N. D. Hatziargyriou, "Optimal distributed generation placement in power distribution networks: Models, methods, and future research," IEEE Transactions on Power Systems, vol. 28 , no. 3, pp. 3420-3428, Aug 2013.

[5] L. Jiang, L. D. Xu, H. Cai, Z. Jiang, F. Bu, and B. Xu, "An iotoriented data storage framework in cloud computing platform," IEEE Transactions on Industrial Informatics, vol. 10, no. 2, pp. 1443-1451, May 2014.

[6] F. Katiraei, R. Iravani, N. Hatziargyriou, and A. Dimeas, "Microgrids management," IEEE Power and Energy Magazine, vol. 6, no. 3, pp. 54-65, May 2008.

[7] M. L. Wymore, J. E. V. Dam, H. Ceylan, and D. Qiao, "A survey of health monitoring systems for wind turbines," Renewable and Sustainable Energy Reviews, vol. 52, pp. 976 - 990, 2015. [Online]. Available: http://www.sciencedirect.com/science/article/pii/S1364032115007571

[8] A. E. Dessler and E. A. Parson, The Science and Politics of Global Climate Change: A Guide to the Debate. Cambridge University Press, 2005.

[9] P. Stott, "How climate change affects extreme weather events," Science, vol. 352, no. 6293, pp. 1517-1518, 2016. [Online]. Available: https://science.sciencemag.org/content/352/6293/1517

[10] M. E. Mann, S. Rahmstorf, K. Kornhuber, B. A. Steinman, S. K. Miller, and D. Coumou, "Influence of anthropogenic climate change on planetary wave resonance and extreme weather events," Scientific Reports, vol. 7, pp. 45242 EP -, Mar 2017, article. [Online]. Available: https://doi.org/10.1038/srep45242

[11] D. P. van Vuuren et al., "Energy, land-use and greenhouse gas emissions trajectories under a green growth paradigm," Global Environmental Change, vol. 42, pp. 237 - 250, 2017. [Online]. Available: http://www.sciencedirect.com/science/article/pii/S095937801630067X

[12] K. Riahi et al., "The shared socioeconomic pathways and their energy, land use, and greenhouse gas emissions implications: An overview," Global Environmental Change, vol. 42, pp. $153-168,2017 . \quad$ [Online]. Available: http://www.sciencedirect.com/science/article/pii/S0959378016300681

[13] C. W. Landsea, "Hurricanes and global warming," Nature, vol. 438, no. 7071, pp. E11-E12, 2005. [Online]. Available: https://doi.org/10.1038/nature04477 
[14] N. Abas, A. Kalair, and N. Khan, "Review of fossil fuels and future energy technologies," Futures, vol. 69, pp. $31-49, \quad 2015 . \quad$ [Online]. Available: http://www.sciencedirect.com/science/article/pii/S0016328715000397

[15] E. Tvinnereim and E. Ivarsflaten, "Fossil fuels, employment, and support for climate policies," Energy Policy, vol. 96, pp. 364 - 371, 2016. [Online]. Available: http://www.sciencedirect.com/science/article/pii/S0301421516302828

[16] T. P. Hughes et al., "Global warming and recurrent mass bleaching of corals," Nature, vol. 543, pp. 373 EP -, Mar 2017, article. [Online]. Available: https://doi.org/10.1038/nature21707

[17] I. Shishlov, R. Morel, and V. Bellassen, "Compliance of the parties to the kyoto protocol in the first commitment period," Climate Policy, vol. 16, no. 6, pp. 768-782, 2016. [Online]. Available: https://doi.org/10.1080/14693062.2016.1164658

[18] J. Rogelj, M. den Elzen, N. Höhne, T. Fransen, H. Fekete, H. Winkler, R. Schaeffer, F. Sha, K. Riahi, and M. Meinshausen, "Paris agreement climate proposals need a boost to keep warming well below 2 c," Nature, vol. 534, pp. 631 EP -, Jun 2016, perspective. [Online] Available: https://doi.org/10.1038/nature18307

[19] M. Jefferson, "Renewable and low carbon technologies policy," Energy Policy, vol. 123, pp. 367 - 372, 2018. [Online]. Available: http://www.sciencedirect.com/science/article/pii/S0301421518304853

[20] E. G. Sakka, D. V. Bilionis, D. Vamvatsikos, and C. J. Gantes, "Onshore wind farm siting prioritization based on investment profitability for greece," Renewable Energy, vol. 146, pp. 2827 - 2839, 2020. [Online]. Available: http://www.sciencedirect.com/science/article/pii/S0960148119312078

[21] I. Monasterolo and M. Raberto, "The impact of phasing out fossil fuel subsidies on the low-carbon transition," Energy Policy, vol. 124, pp. 355 - 370, 2019. [Online]. Available: http://www.sciencedirect.com/science/article/pii/S0301421518305809

[22] S. Pfenninger, J. DeCarolis, L. Hirth, S. Quoilin, and I. Staffell, "The importance of open data and software: Is energy research lagging behind?" Energy Policy, vol. 101, pp. 211 - 215, 2017. [Online]. Available: http://www.sciencedirect.com/science/article/pii/S0301421516306516

[23] B. Chen, Y. Yang, and Z. L. Wang, "Scavenging wind energy by triboelectric nanogenerators," Advanced Energy Materials, vol. 8 , no. 10, p. 1702649,2018 . [Online]. Available: https://onlinelibrary.wiley.com/doi/abs/10.1002/aenm.201702649

[24] S. Mujeeb, T. A. Alghamdi, S. Ullah, A. Fatima, N. Javaid, and T. Saba, "Exploiting deep learning for wind power forecasting based on big data analytics," Applied Sciences, vol. 9, no. 20, 2019. [Online]. Available: https://www.mdpi.com/2076-3417/9/20/4417

[25] Y. Li, B. Feng, G. Li, J. Qi, D. Zhao, and Y. Mu, "Optimal distributed generation planning in active distribution networks considering integration of energy storage," Applied Energy, vol. 210, pp. 1073 - 1081, 2018. [Online]. Available: http://www.sciencedirect.com/science/article/pii/S0306261917310231

[26] X. Zhao, S. Wang, and T. Li, "Review of evaluation criteria and main methods of wind power forecasting," Energy Procedia, vol. 12, pp. 761 - 769, 2011, the Proceedings of International Conference on Smart Grid and Clean Energy Technologies (ICSGCE 2011. [Online]. Available: http://www.sciencedirect.com/science/article/pii/S187661021101928X

[27] J. Yan, F. Li, Y. Liu, and C. Gu, "Novel cost model for balancing wind power forecasting uncertainty," IEEE Transactions on Energy Conversion, vol. 32, no. 1, pp. 318-329, March 2017.

[28] G. Santamar'ia-Bonfil, A. Reyes-Ballesteros, and C. Gershenson, "Wind speed forecasting for wind farms: A method based on support vector regression," Renewable Energy, vol. $85, \quad$ pp. $790-809,2016 . \quad$ [Online]. Available: http://www.sciencedirect.com/science/article/pii/S0960148115301014

[29] D. Silver et al., "Mastering the game of go with deep neural networks and tree search," Nature, vol. 529, pp. 484 EP -, Jan 2016, article. [Online]. Available: https://doi.org/10.1038/nature16961

[30] D. Tayal, "Achieving high renewable energy penetration in western australia using data digitisation and machine learning," Renewable and Sustainable Energy Reviews, vol. 80, pp. 1537 - 1543, 2017. [Online]. Available: http://www.sciencedirect.com/science/article/pii/S1364032117311097

[31] "Kite power: No longer a flight of fancy?" https://www.shell.com/inside-energy/kite-power.html, accessed: 2019-11-07.
[32] "Google renewable envergy global projects," https://about.google/intl/en-GB/stories/renewable-energy-is-boostingeconomies/, accessed: 2019-11-07.

[33] "Deepmind," https://deepmind.com/blog/article/machine-learning-canboost-value-wind-energy, accessed: 2019-11-07.

[34] "Advanced intelligence for energy markets." https://advmicrogrid.com/, accessed: 2019-11-07.

[35] R. G. Kavasseri and K. Seetharaman, "Day-ahead wind speed forecasting using f-arima models," Renewable Energy, vol. 34, no. 5, pp. 1388 - 1393, 2009. [Online]. Available: http://www.sciencedirect.com/science/article/pii/S0960148108003327

[36] A. M. Foley, P. G. Leahy, A. Marvuglia, and E. J. McKeogh, "Current methods and advances in forecasting of wind power generation," Renewable Energy, vol. 37, no. 1, pp. 1 - 8, 2012. [Online]. Available: http://www.sciencedirect.com/science/article/pii/S0960148111002850

[37] H. Holttinen, "Optimal electricity market for wind power," Energy Policy, vol. 33, no. 16, pp. 2052 - 2063, 2005. [Online]. Available: http://www.sciencedirect.com/science/article/pii/S0301421504001016

[38] J. Wang, S. Qin, Q. Zhou, and H. Jiang, "Mediumterm wind speeds forecasting utilizing hybrid models for three different sites in xinjiang, china," Renewable Energy, vol. 76, pp. $91-101,2015 . \quad$ [Online]. Available: http://www.sciencedirect.com/science/article/pii/S0960148114007265

[39] E. Cadenas, O. Jaramillo, and W. Rivera, "Analysis and forecasting of wind velocity in chetumal, quintana roo, using the single exponential smoothing method," Renewable Energy, vol. 35, no. 5, pp. 925 - 930, 2010. [Online]. Available: http://www.sciencedirect.com/science/article/pii/S0960148109004765

[40] O. A. Jaramillo and M. A. Borja, "Bimodal versus weibull wind speed distributions: An analysis of wind energy potential in la venta, mexico," Wind Engineering, vol. 28, no. 2, pp. 225-234, 2004. [Online]. Available: https://doi.org/10.1260/0309524041211404

[41] O. Jaramillo and M. Borja, "Wind speed analysis in la ventosa, mexico: a bimodal probability distribution case," Renewable Energy, vol. 29 , no. 10 , pp. 1613 - 1630, 2004. [Online]. Available: http://www.sciencedirect.com/science/article/pii/S0960148104000618

[42] M. Jafarian and A. Ranjbar, "Fuzzy modeling techniques and artificial neural networks to estimate annual energy output of a wind turbine," Renewable Energy, vol. 35, no. 9, pp. $2008-2014,2010 . \quad$ [Online]. Available: http://www.sciencedirect.com/science/article/pii/S0960148110000509

[43] I. Segura-Heras, G. Escrivá-Escrivá, and M. Alcázar-Ortega, "Wind farm electrical power production model for load flow analysis," Renew able Energy, vol. 36, no. 3, pp. 1008 - 1013, 2011. [Online]. Available: http://www.sciencedirect.com/science/article/pii/S0960148110004192

[44] E. Cadenas and W. Rivera, "Wind speed forecasting in the south coast of oaxaca, méxico," Renewable Energy, vol. 32, no. 12, pp. $2116-2128$, 2007. [Online]. Available: http://www.sciencedirect.com/science/article/pii/S0960148106002801

[45] K. A. Larson and K. Westrick, "Short-term wind forecasting using off-site observations," Wind Energy, vol. 9, no. 1-2, pp. 55-62, 2006. [Online]. Available: https://onlinelibrary.wiley.com/doi/abs/10.1002/we.179

[46] A. Paniagua-Tineo, S. Salcedo-Sanz, C. Casanova-Mateo, E. Ortiz-García, M. Cony, and E. Hernández-Martín, "Prediction of daily maximum temperature using a support vector regression algorithm," Renewable Energy, vol. 36, no. $11, \quad$ pp. 3054 - 3060, 2011. [Online]. Available: http://www.sciencedirect.com/science/article/pii/S0960148111001443

[47] J. Zeng and W. Qiao, "Short-term solar power prediction using a support vector machine," Renewable Energy, vol. 52, pp. 118 - 127, 2013. [Online]. Available: http://www.sciencedirect.com/science/article/pii/S0960148112006465

[48] J. Hu, J. Wang, and G. Zeng, "A hybrid forecasting approach applied to wind speed time series," Renewable Energy, vol. 60, pp. $185-194$, 2013. [Online]. Available: http://www.sciencedirect.com/science/article/pii/S0960148113002577

[49] "Short-term wind speed forecasting using wavelet transform and support vector machines optimized by genetic algorithm," Renewable Energy, vol. 62, pp. 592 - 597, 2014.

[50] T. Kleynhans, M. Montanaro, A. Gerace, and C. Kanan, "Predicting top-of-atmosphere thermal radiance using merra-2 atmospheric data with deep learning," Remote Sensing, vol. 9, no. 11, 2017. [Online]. Available: https://www.mdpi.com/2072-4292/9/11/1133 